\title{
Determination of Soluble Sugars in Arabidopsis thaliana Leaves by Anion Exchange Chromatography
}

M. Isabel Ortiz-Marchena, M. Teresa Ruiz, Federico Valverde and José M. Romero*

Plant Development Unit. Instituto de Bioquímica Vegetal y Fotosíntesis, Consejo Superior de Investigaciones Científicas and Universidad de Sevilla, Seville, Spain

*For correspondence: imromero@us.es

[Abstract] Determination of soluble sugars is basic for the study of carbon metabolism in plants. Soluble sugar quantitation can be achieved by enzymatic methods implying different coupled reactions. Here we describe a simple method that allows rapid determination of the most abundant soluble sugars (glucose, fructose and sucrose) in Arabidopsis leaves by anion exchange chromatography. We have applied this method to study the levels of soluble sugars during the photoperiodic transition to flowering (Ortiz-Marchena et al., 2014).

\section{Materials and Reagents}

1. Plants grown in soil for 3 weeks

Note: Treatment of samples is explained in the Procedure section.

2. Liquid $\mathrm{N}_{2}$

3. Absolute ethanol

4. HEPES (Sigma-Aldrich, catalog number: H4034-1KG)

5. $\mathrm{KOH}$ (Panreac Applichem, catalog number: 121515)

6. $\mathrm{NaOH} 50 \%(w / v)$ solution (AppliChem $\mathrm{GmbH}$, catalog number: A3720, 1000)

7. Milli Q grade water

8. $100 \mathrm{mM}$ HEPES-KOH (see Recipes)

9. Extraction buffer 1 (EB1) (see Recipes)

10. $0.1 \mathrm{M} \mathrm{NaOH}$ (see Recipes)

11. $21.6 \mathrm{mM} \mathrm{NaOH}$ (see Recipes)

\section{Equipment}

1. Small mortar and pestle

2. $1.5 \mathrm{ml}$ microfuge tubes

3. Automatic pipettes

4. Precision scale 
5. $2 \mathrm{ml}$ microcentrifuge (Eppendorf, model: 5415R)

6. Vacufuge concentrator 5301 (Eppendorf)

7. Nylon filters (Whatman, catalog number: UN203NPENYL)

8. Dionex HPLC system (Dionex ICS 5000)

9. CarboPacPA10 column ( $4 \times 250 \mathrm{~mm}$ ) (Thermo Fisher Scientific, catalog number: 046110)

10. CarboPacPA10 Pre-column ( $450 \mathrm{~mm}$ ) (Thermo Fisher Scientific, catalog number: 046115)

11. Gold electrode (Dionex ICS 5000)

12. Computer connected to HPLC system

\section{Software}

1. Chromeleon software (v.7.0) (Thermo Fisher Scientific)

\section{Procedure}

1. Collect leaf sample (or plant material) and freeze immediately in liquid $\mathrm{N}_{2}$. Stored at -80 ${ }^{\circ} \mathrm{C}$ until use.

2. Grind plant tissue with mortar and pestle in the presence of liquid $N_{2}$ until the sample is converted to a fine powder.

3. Weigh 100-300 mg leaf powder into a microfuge tube and add extraction buffer 1 . [The ratio of powered tissue/EB1 should be 1:1 (w/v).]

4. Heat the samples at $80{ }^{\circ} \mathrm{C}$ for $2 \mathrm{~h}$ to extract soluble sugars. Allow them to cool and centrifuge at $15,000 \times g(13,000 \mathrm{rpm}$ in a microfuge $) 10 \mathrm{~min}$.

5. Remove supernatant to a fresh microfuge tube.

6. The supernatant is evaporated in vacuum at $45^{\circ} \mathrm{C}$ for $2 \mathrm{~h}$.

7. Resuspend the dried extract in Milli $Q$ grade water. [The ratio of water/original powdered tissue should be 0.5:1 (v/w).]

8. Keep on ice while preparing all samples. Freeze the samples at $-20{ }^{\circ} \mathrm{C}$ for long storage, when necessary.

9. Clean soluble plant extracts by filtering through nylon filters. Suitable extract volumes (typically $50 \mu \mathrm{l}$ ) were made up to $200 \mu \mathrm{l}$ with Milli Q grade water and filtered through Whatman Mini-Uniprep ${ }^{\mathrm{TM}}$ nylon filters $(0.2 \mu \mathrm{m}$ pore size).

a. Add $250 \mu \mathrm{l} 21.6 \mathrm{mM} \mathrm{NaOH}$ to $50 \mu \mathrm{l}$ filtered extracts and apply into a Dionex HPLC system. Set a $10 \mu$ injection volume.

b. Sugars are separated by isocratic elution at $20{ }^{\circ} \mathrm{C}$ with $18 \mathrm{mM} \mathrm{NaOH}$ as mobile phase at a flow of $1 \mathrm{ml} / \mathrm{min}[18 \%(\mathrm{v} / \mathrm{v}) 0.1 \mathrm{M} \mathrm{NaOH}$ and $82 \%(\mathrm{v} / \mathrm{v})$ Milli $\mathrm{Q}$ grade 
water] through a CarboPac PA10 column and pre-column CarboPacPA10. Detection is carried out by amperometric detection with a gold electrode in $\mathrm{nC}$ units.

c. The chromatograms are analysed using the Chromeleon software. Peaks are identified by their retention time in comparison with known standard sugars. The peak area is calculated by integrating the area between the start and the end of a peak (nC $x$ min) using the Chromeleon software. A range of sugar mixtures of known concentrations $(1,0.5,0.1,0.05$ and $0.01 \mathrm{mM}$; as an example, see the $0.5 \mathrm{mM}$ standard profile in Figure $1 \mathrm{~A}$ ) is applied into the HPLC system, as described above, to generate calibration curves for each sugar, relating peak area (as determined by the software) with concentration. The amount of a certain sugar is calculated comparing its peak area (Figure 1B) with those in the calibration curves. Sugar content in samples is expressed in micromoles of sugar per gram fresh weight.
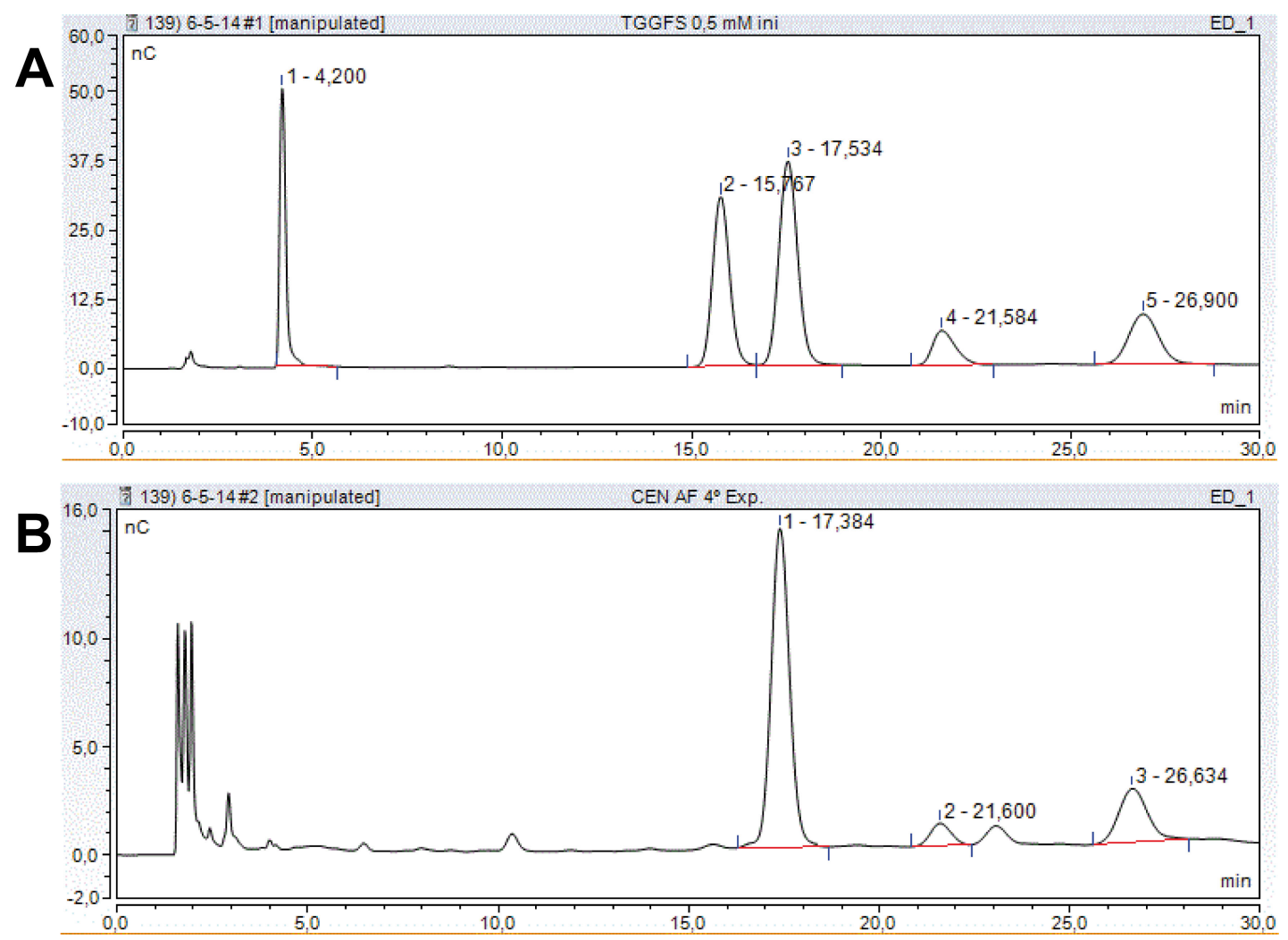

Figure 1. Sugar elution profiles in typical chromatograms. A. Sugar standards: Mixture of trehalose (peak 1), galactose (peak 2), glucose (peak 3), fructose (peak 4) and sucrose (peak 5) at $0.5 \mathrm{mM}$ each. B. Plant extract showing glucose (1), fructose (2) and sucrose (3) peaks. Retention times are indicated for each peak. $\mathrm{nC}: 10^{-9} \mathrm{C}$. 


\section{Representative data}

1. Between working days and during weekends, columns and pre-columns were washed with $0.2 \mathrm{M} \mathrm{NaOH}$ at $0.1 \mathrm{ml} \mathrm{min}^{-1}$ flow. After this treatment, they were equilibrated with 18 $\mathrm{mM} \mathrm{NaOH}$ at $1 \mathrm{ml} / \mathrm{min}$ flow for at least one hour prior to sample loading. In order to monitor the reproducibility of the different analysis, aliquots of the $0.5 \mathrm{mM}$ sugar standard mixture were analysed, as mentioned above, at the beginning and at the end of each sample batch.

Table 1. Typical area values for the glucose, fructose and sucrose peaks in the $\mathbf{0 . 5}$ $\mathrm{mM}$ sugar standard aliquots $(n=18)$

\begin{tabular}{|c|c|c|c|}
\hline Area (nC $\times$ min) & Glucose & Fructose & Sucrose \\
\hline Mean & 20.5 & 4.3 & 8.4 \\
\hline SD & 1.2 & 0.4 & 0.9 \\
\hline
\end{tabular}

The peak area values from a representative plant extract (i.e. Figure 1B) were 8.3, 0.5 and $2.2 \mathrm{nC} \times$ min for glucose, fructose and sucrose, respectively. These values could differ among samples, as expected since they were obtained in different experimental conditions, but they always fell into the measurable range corresponding to the calibration curve.

\section{$\underline{\text { Notes }}$}

1. The use of Dionex equipment (or similar chromatographers containing plastic connections and tubing) is mandatory, as regular HPLC systems are not compatible with $\mathrm{NaOH}$ solutions.

2. A helium stream should be used in order to degasify the mobile phase.

\section{$\underline{\text { Recipes }}$}

1. $100 \mathrm{mM}$ HEPES-KOH $(\mathrm{pH} 7.7)$

Weigh $23.83 \mathrm{~g}$ HEPES

Adjust $\mathrm{pH}$ to 7.7 with $\mathrm{KOH}$

Add Milli Q grade water to $1 \mathrm{~L}$

Stored at $4{ }^{\circ} \mathrm{C}$

2. Extraction buffer 1 (EB1)

Mix 20 ml 100 mM HEPES-KOH (pH 7.7) with 80 ml EtOH 100\% (v/v) 
Stored at RT

3. $0.1 \mathrm{M}$ and $0.2 \mathrm{M} \mathrm{NaOH}$

Dilute $5.25 \mathrm{ml}$ or $10.5 \mathrm{ml}$ (respectively) $\mathrm{NaOH} 50 \%$ (w/v) with MilliQ grade water to $1 \mathrm{~L}$

Stored at RT for no longer than 2 weeks due to carbonation

4. $21.6 \mathrm{mM} \mathrm{NaOH}$

Dilute $2.16 \mathrm{ml} 0.1 \mathrm{M} \mathrm{NaOH}$ with Milli Q grade water to $10 \mathrm{ml}$

Stored at RT for no longer than 2 weeks due to carbonation

\section{Acknowledgments}

This work was performed with funding from projects CSD2007-00057, BIO2008-02292, and BIO2011-28847-C02-00 (Spanish Ministry of Economy and Competitiveness, MINECO) and Excellence projects P06-CVI-01450 and P08-AGR-03582 (Junta de Andalucía) partially supported by FEDER funding to F.V. and J.M.R. We also acknowledge the TRANSPLANTA consortium, Project CONSOLIDER 28317 (MINECO).

\section{References}

1. Ortiz-Marchena, M. I., Albi, T., Lucas-Reina, E., Said, F. E., Romero-Campero, F. J., Cano, B., Ruiz, M. T., Romero, J. M. and Valverde, F. (2014). Photoperiodic control of carbon distribution during the floral transition in Arabidopsis. Plant Cell 26(2): 565-584. 